\section{Atmosphärendruckplasma zur funktionalen Pulverbeschichtung}

Bei ihrem ersten gemeinsamen Messeauftritt werden die B Kooperationspartner Plasmatreat und EFC-Plasma auf dem Gemeinschaftsstand SurfPlaNet eine neu entwickelte Anlage zur Feinpulverbeschichtung unter Einsatz von Atmosphärendruckplasma präsentieren. Basis ist die von Plasmatreat entwickelte Plasmadüsentechnologie Openair. Das sogenannte FPC (Fine Powder Coating)-Plasmaverfahren dient der ortsselektiven, homogenen und funktionalen Metallisierung und Feinbeschichtung von vornehmlich Kunststoffen und Metall, aber auch Glas und Keramik. Die Technologie zeichnet sich durch ihre Inline-Fähigkeit, hohen Prozessgeschwindigkeit und millimetergenaue Auftragsmöglichkeit aus. Dem neuen Beschichtungsprozess dient das „kalte Plasma" nicht nur zur mikrofeinen Reinigung und hohen Aktivierung der Materialoberflächen, sondern gleichermaßen als sicheres und schnelles Transportmedium. Das Pulver wird direkt in den Plasmastrahl eingegeben und von diesem auf die Oberfläche des Substrats befördert, wo es die vom Anwender gewünschte Funktionsschicht bildet. Entscheidend für den Erfolg der richtigen Beschichtung ist, dass Pulver, Pulverförderung und Plasmatechnologie zu 100\% zusammenpassen.

www.plasmatreat.de, www.efc-plasma.de

Halle 3, Stand F 43

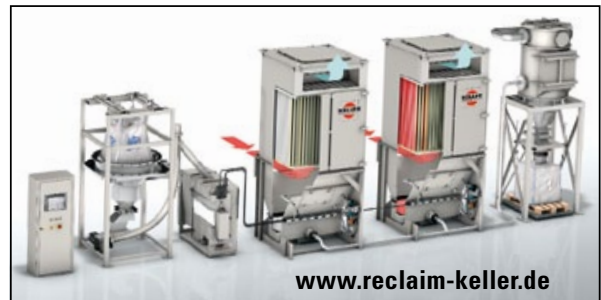

Nass lackieren - Trocken abscheiden regenerierbar - energieeffizient - nachhaltig

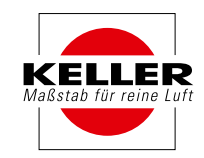

